\title{
Night-time F-region and daytime E-region ionospheric drifts measured at Udaipur during solar flares
}

\author{
B. M. Vyas and R. Pandey \\ Department of Physics, M. L. Sukhadia University, Udaipur 313 001, India \\ Received: 19 December 2001 - Revised: 15 April 2002 - Accepted: 27 May 2002
}

\begin{abstract}
Ionospheric drifts measured at a low latitude station, Udaipur (Geomag. Lat. $14.5^{\circ} \mathrm{N}$ ), in the night-time Fregion and daytime E-region during solar flares have been studied. The night-time observations, which correspond to the F-region drifts, were carried out on five different nights. The daytime observation corresponding to the E-region drifts is only for one day. It is found that the apparent drift during the solar flare period is reduced considerably, in the daytime E-region as well as in the night-time F-region. The East-West and North-South components of the apparent drift speed are also similarly affected. For the daytime E-region drifts during a flare, increased ionization and subsequent reduction of reflection height is proposed to be the cause of reduced drift speeds. For the night-time F-region drifts, a reduced electric field at the F-region heights resulting from coupling of sunlit and dark hemispheres has been proposed to be the possible cause.
\end{abstract}

Key words. Ionosphere (electric fields and currents; ionospheric disturbances)

\section{Introduction}

The closely spaced receiver technique (Mitra, 1949) has been employed extensively for the measurement of ionospheric horizontal drift at high latitudes (e.g. Briggs and Spencer, 1954; Patel and Chandra, 1982), equatorial latitudes (Skinner et al., 1963; Rastogi et al., 1972; Vyas and Chandra 1979), and low latitudes (Rastogi et al., 1978; Patel and Chandra 1980; Vyas 1989). These studies have given rise to a fair understanding of various morphological features, including seasonal variations of the horizontal ionospheric drifts. Effects of solar and magnetic activity have also been studied extensively. It is well known that the Sun is the main driving force of a vast majority of ionospheric phenomena, including the sustenance of the ionosphere. A disturbed Sun therefore has an overbearing influence on the ionospheric dynamics.

Correspondence to: R. Pandey (pandey_r@yahoo.com)
A disturbed Sun produces both prompt and delayed effects in the ionosphere. While the delayed effects are more commonly associated with the ionospheric storms, the prompt effects are attributed to the solar flares (Buonsanto 1999). One of the prompt effects of solar flares is the disturbance in the geomagnetic field. This has been studied by several workers (Mc Nish 1937; Nagata 1966; Forbush and Casaverde 1961; Rastogi et al., 1975).

In spite of a large number of studies on ionospheric drifts, under a variety of conditions, ones during solar flares are rather rare. This is particularly so in case of the low and tropical latitudes. The effect of solar flares on night-time ionospheric drifts has not been reported in the literature. Hence the present study aims to assess the effect of solar flares on ionospheric drifts at a low latitude station, namely, Udaipur, (Geog. Lat., 24.6 ${ }^{\circ} \mathrm{N}$, Geog. Long., 73.4 E, Geomag. Lat. $\left.14.5^{\circ} \mathrm{N}\right)$, India.

Ionospheric drift measurements, made during the year 1981-82, have been used in the present study. The drift records were analyzed using the similar fade (or time delay) method (e.g. Vyas 1989) for obtaining the apparent drift speed and its direction. The period of some of the observations coincided with the occurrence of solar flares, verified from the solar geophysical data supplied by the National Physical laboratory, New Delhi, India. It is well known that the daytime drift observations during intense solar flares are obscured due to increased non-deviative absorption in the region underlying reflection points. Hence the drift record during the daytime flare at Udaipur is the only one available in this data set, and could be obtained only during the morning hours when the plasma density is normally low. The night-time observations coincided with the occurrence of solar flares effects at other longitudes.

\section{Experimental details}

The closely spaced receiver technique is based on illuminating the ionosphere by radio wave pulses with a frequency 
24 April 1981
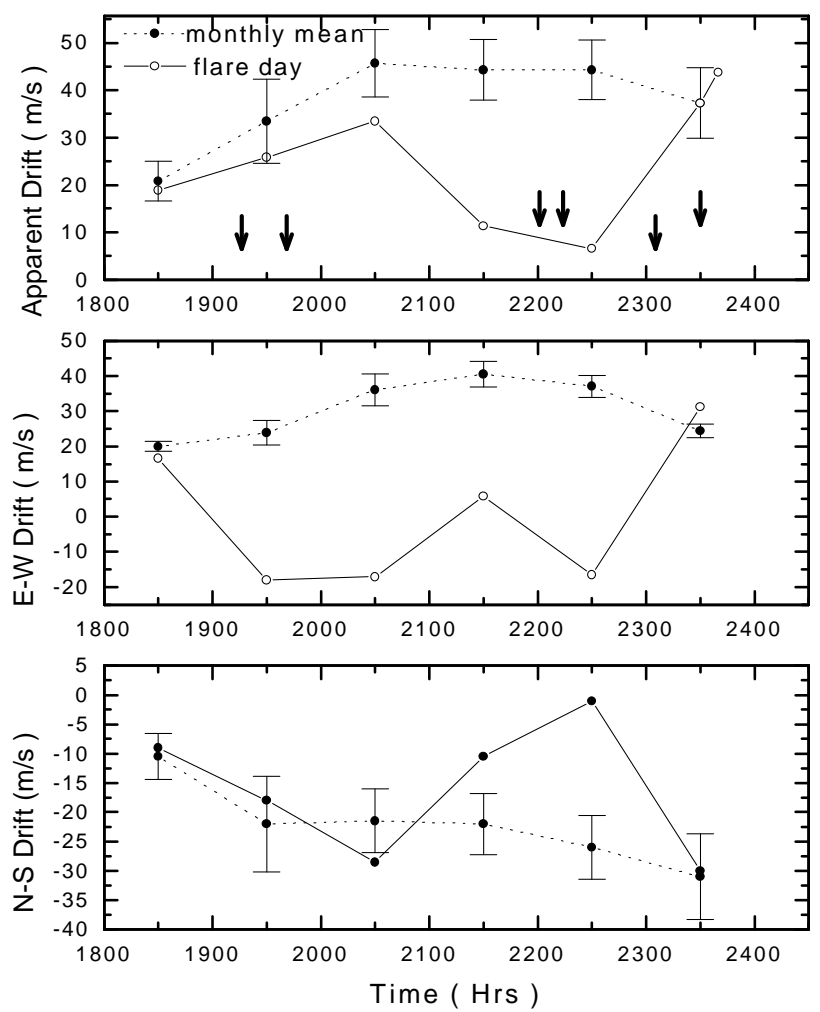

Fig. 1. Variation of Apparent Drift Speed, its E-W and N-S components on 24 April 1981, along with their respective monthly mean variation. Solid circles connected by dotted lines represent the monthly mean variation. Open circles connected by solid lines represent the speeds on the flare day. Downward pointing arrows from the same ordinate level represent the time of onset, maximum intensity and end of a flare. Solar flares occurred at 19:16, 19:41 and 23:05 (2B) and 22:01, 22:14 and 23:30 (1B).

of a few MHz. The amplitude pattern of the reflected radio wave is recorded simultaneously at three points on the ground, which are situated at the vertices of an isosceles right-angled triangle, separated by a distance that is of the order of the wavelength of the transmitted radio wave.

The experimental arrangement of this technique consists of a radio frequency pulsed transmitter, a transmitting antenna, a receiving and recording system. The transmitter generates pulses of radio frequency of $2.5 \mathrm{MHz}$ having a peak power of $5 \mathrm{~kW}$. The pulses, with a repetition frequency of $50 \mathrm{~Hz}$, are generated having a variable pulse width between 100 to $300 \mu \mathrm{s}$. The transmitting as well as the receiving antennae are half-wave dipoles. The receiving antennae are placed at the corners of an isosceles right-angled triangle with sides measuring $90 \mathrm{~m}, 90 \mathrm{~m}$, and $127 \mathrm{~m}$. The signals from the antennae are brought to the receiving unit and successively connected to a single receiver by means of an electronic switch. The switch generates three step waves which are applied to the recording oscilloscope giving three spots corresponding to the three antennae. The amplitude variations of the reflected radio wave at the three closely
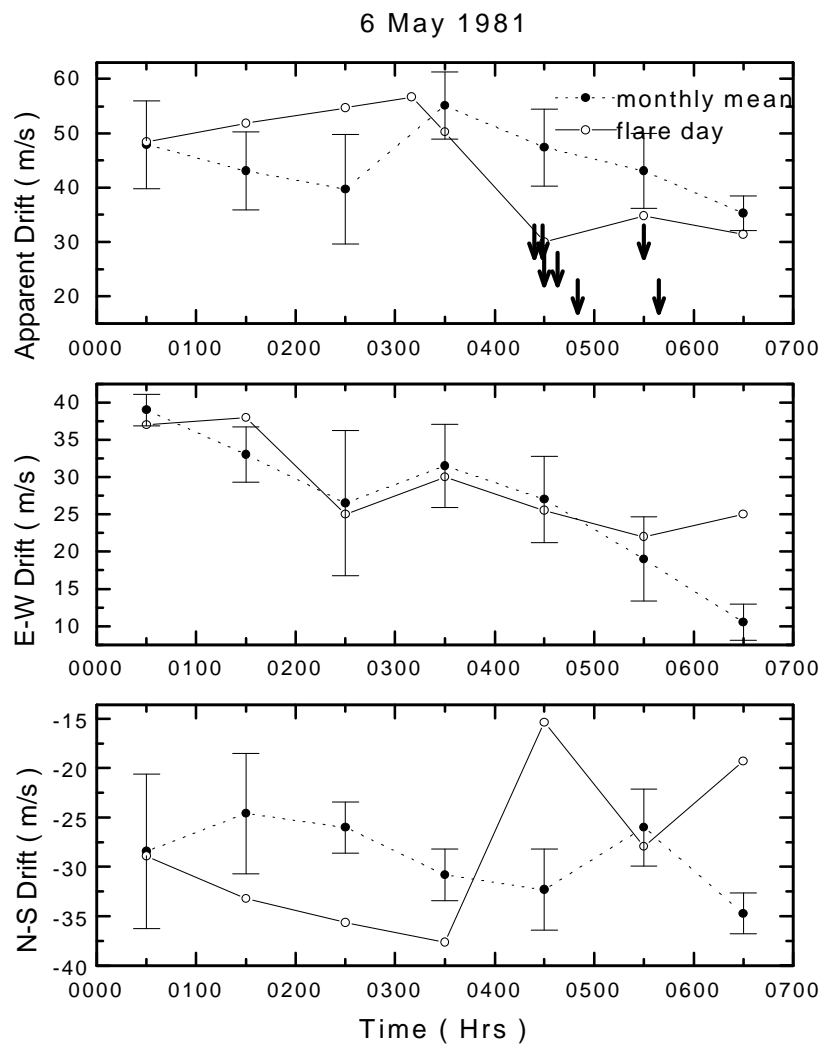

Fig. 2. Variation of Apparent Drift Speed, its E-W and N-S components on 6 May 1981, along with their respective monthly mean variation. Solar flares occurred at 04:24, 04:29 and 05:30 (2B), 04:30, 04:38 and 05:30 (1N), and 04:50 and 05:39 (1F). The rest of the details are the same as in Fig. 1.

spaced aerials are displayed on the cathode ray screen and are recorded on $35 \mathrm{~mm}$ film moving at speed of $5 \mathrm{~cm} / \mathrm{min}$. A time marker unit generates a mark every $12 \mathrm{~s}$ that is also recorded on the film.

The temporal variation of the amplitude of the reflected radio waves is also known as fading. This arises due to the horizontal movement of the ionization irregularities that are embedded in the medium and drift along with the background plasma. By recording the fading at the three points on the ground, the speed and direction of motion of the ionospheric irregularities is extracted. This is done by measuring the time delays between the positions of the maxima observed at the two sets of antennae and their distances. This method of analysis is also known as the similar fade method. Further details of the experimental arrangement and method of analysis have been described elsewhere ( e.g. Rastogi et al., 1978; Vyas, 1989).

\section{Results}

Temporal variation of apparent drift speed along with its East-West and North-South components on six different days when solar flares occurred, is given in Figs. 1-6 as curves 


\begin{tabular}{cccccc}
\hline Night-time & Date & Time & Flare type \\
& 24 April 1981 & $19: 16$ & $19: 41$ & $23: 05$ & $2 \mathrm{~B}$ \\
& & $22: 01$ & $22: 14$ & $23: 30$ & $1 \mathrm{~B}$ \\
& \multirow{2}{*}{ 6 May 1981 } & $04: 24$ & $04: 29$ & $05: 30$ & 2B \\
& & $04: 30$ & $04: 38$ & $05: 30$ & $1 \mathrm{~N}$ \\
& & $04: 50$ & - & $05: 39$ & $1 \mathrm{~F}$ \\
& 24 July 1981 & $22: 05$ & $22: 09$ & $22: 50$ & $1 \mathrm{~B}$ \\
& 10 August 1982 & $20: 31$ & $20: 55$ & $22: 01$ & $2 \mathrm{~N}$ \\
& & $23: 34$ & - & $23: 48$ & $1 \mathrm{~N}$ \\
& 2 November 1982 & $21: 50$ & - & $22: 45$ & $1 \mathrm{~N}$ \\
\hline Daytime & 15 September 1982 & $07: 25$ & $07: 51$ & $08: 21$ & $1 \mathrm{~B}$ \\
\hline
\end{tabular}

24 July 1981
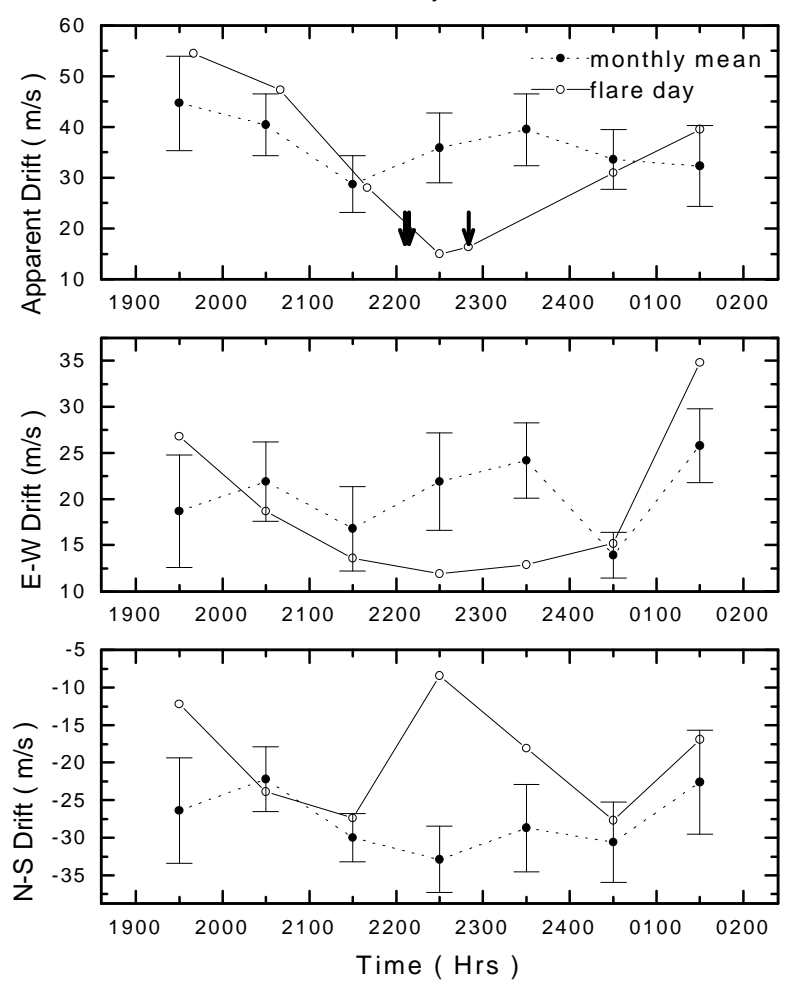

Fig. 3. Variation of Apparent Drift Speed, its E-W and N-S components on 24 July 1981, along with their respective monthly mean variation. Solar flare occurred at 22:05, 22:09 and 22:50 (1B). The rest of the details are the same as in Fig. 1.

with open circles connected by full lines. The solid circles connected by dashed lines represent the curves for monthly mean variations, obtained by excluding the flare days; these are also shown in the figures. The normal variability of the drifts, in the absence of a solar flare, is shown by plotting the standard deviation as error bars on the monthly mean trace in all the figures. A negative value for the East-West and North-South components implies a westward and southward direction, respectively. The local time (LT) in hh:mm format
10 August 1982
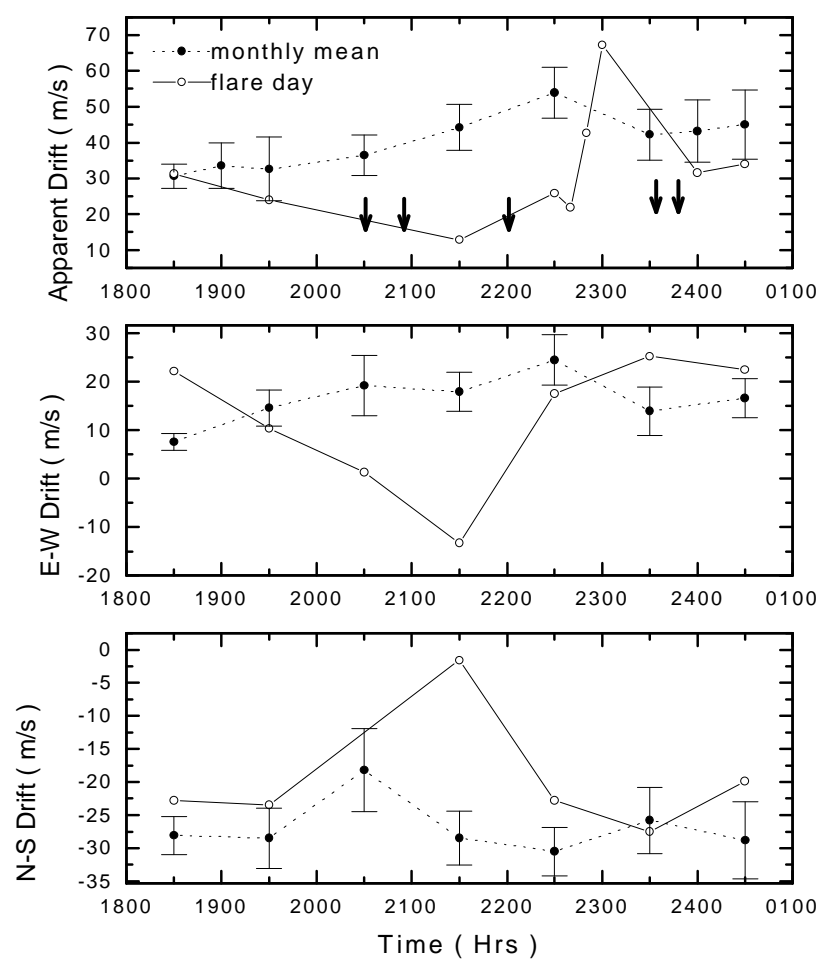

Fig. 4. Variation of Apparent Drift Speed, its E-W and N-S components on 10 August 1982, along with their respective monthly mean variation. Solar flares occurred at 20:31, 20:55 and 22:01 (2N), and 23:34 and 23:48 (1N). The rest of the details are the same as in Fig. 1.

is the abscissa for these curves. The time of occurrence of the solar flares is indicated by downward pointing vectors. Normally, a flare is characterized by three phases, namely, onset, maximum and its end. Thus, the vectors starting from the same ordinate level represent the corresponding phases. Table 1 gives a list of the flare days on which observations are available. The table also gives the period of a flare and its type. On some of the days, more than one flare was reported. 
2 November 1982
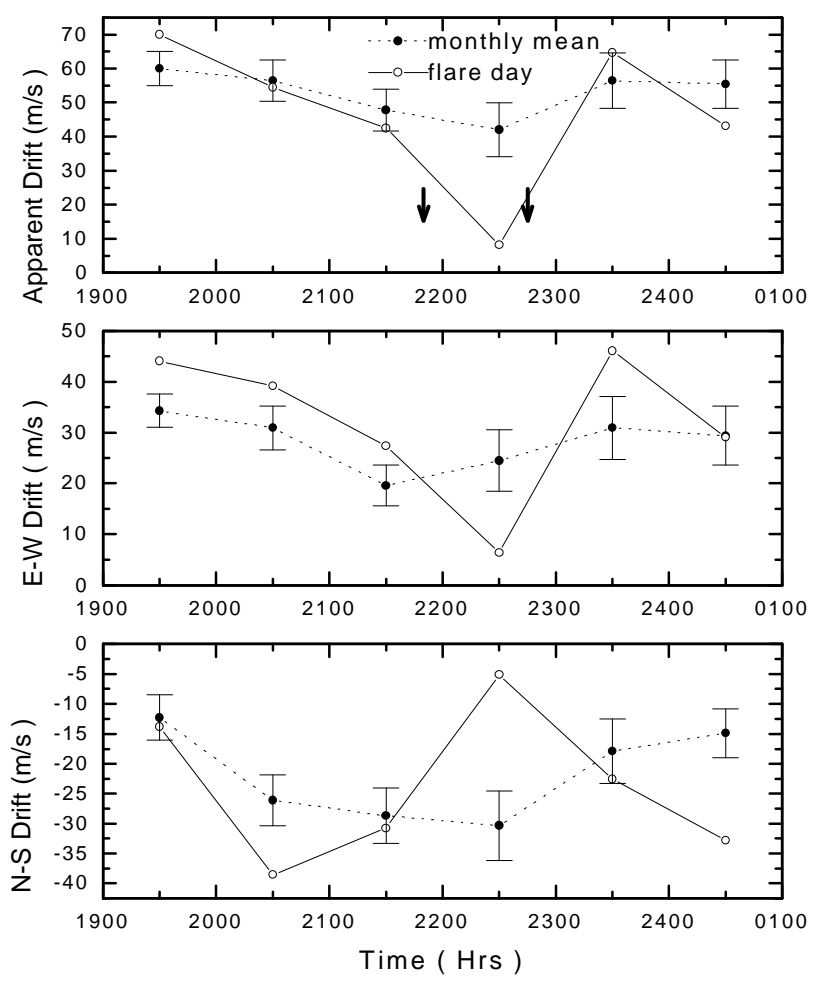

Fig. 5. Variation of Apparent Drift Speed, its E-W and N-S components on 2 November 1982, along with their respective monthly mean variation. The solar flare occurred at 21:50 and 22:45 (1N). The rest of the details are the same as in Fig. 1.

\subsection{Night-time cases}

Results for the night-time cases are shown in Figs. 1-5. As noted earlier, these observations correspond to the periods when the flares were observed at other sunlit longitudes. Figure 1 gives results for the night of 24 April 1981 when two flares were reported. The first flare had its onset at 19:16 LT, reached its maximum at 19:41 LT and was over at 23:05 LT. While the first flare was in its declining phase, another flare started at 22:01 LT, reached its maximum at 22:14 LT and was over at 23:30 LT. Thus, while the onset and maximum of the first flare occurred in the evening twilight hours, the second flare occurred during the night hours. The top panel in Fig. 1 gives the variation of apparent drift speed with local time. The East-West and North-South components of the apparent drift speed are also plotted in this figure. It can be seen from Fig. 1 that the apparent drift speed decreases from its normal value soon after the onset of the flare. With the termination of the flare, the drift speed tends to return to its normal value. During the major part of the flare, the drift speed is significantly lower than the normal variability of the drift speeds. A very pronounced effect of the solar flare is evident in the East-West component of the apparent drift where, not only the speed is reduced, but its direction is also reversed for a major part of the solar flare. Prominent changes in the
15 September 1982
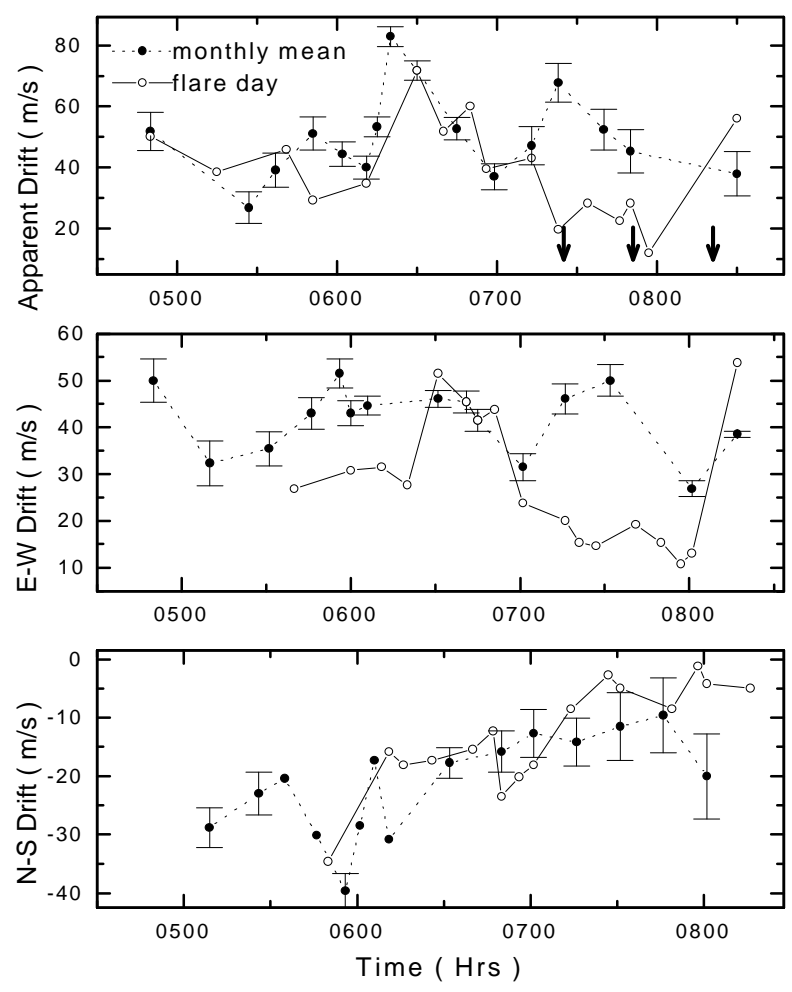

Fig. 6. Variation of Apparent Drift Speed, its E-W and N-S component during the daytime on 15 September 1982, along with their respective monthly mean variation. The solar flare occurred at 07:25, 07:51 and 08:21 (1B). The rest of the details are the same as in Fig. 1.

North-South component of the apparent drift are discernible only during the second flare. Since the North-South component of the drift speed at Udaipur is normally southward, its less negative value implies a reduction in its value.

Figure 2 shows the results for 6 May 1981. On this day, three different types of flares occurred prior to sunrise. It is obvious from the figure that the apparent drift speed decreases significantly during the solar flare. While the EastWest component does not show any significant reduction, the North-South component does.

Figure 3 shows the results for 24 July 1981. Only one solar flare, Type B, occurred on this night, well past sunset. The two downward pointing vectors, indicating the onset and maximum of the flare, have merged into one due to the small time difference between the two. A significant reduction in apparent drift speed during the flare period is clearly seen. Also, both the East-West and North-South components show reduced drift speeds corresponding to the flare period. The drift speed tends to return to its normal value after the flare is terminated.

Figure 4 shows the results for 10 August 1982. Two Ntype flares occurred on this night after 20:30 LT. The flare periods are separated by about one hour and a half. It can be seen from Fig. 4 that the apparent drift speed decreased 
significantly with the onset of the first flare at 20:31 LT. With the termination of this flare, the apparent drift speed reached its normal value. The effect is not significant during the second flare. It is probably due to the short duration of the flare, which was less than $15 \mathrm{~min}$. Noticeable reduction in both the components of the drift speed during the first flare period is clearly seen.

The night of 2 November 1982 had only one flare. Results for this night are given in Fig. 5. A clear-cut reduction in the apparent drift speed and its components during the flare period is evident from this figure. Again, with the termination of the flare, the apparent drift speed tended to return to its normal value.

\subsection{Daytime case}

As has been noted earlier, daytime observations during solar flares are rarer due to increased non-deviative absorption. Hence the data is limited to just one daytime observation. Results for this day are shown in Fig. 6. On this day, only one flare occurred in the morning hours. As was observed for the night-time cases, during the daytime also, the apparent drift speed is seen to decrease with the occurrence of the flare. A similar decrease in its East-West component is evident from the figure.

\section{Discussion}

From the foregoing results, it can be concluded that the ionospheric drift speed during a solar flare is reduced considerably, with respect to its monthly mean value as well as to the prevalent value prior to the onset of the flare. The reduction in the drift speed nearly coincides with the onset of the flare. The effect of solar flares on ionospheric E- and F-region drift speed during daytime for an equatorial station, Thumba, has been reported by Misra (1971), who found that the drift speed reduces during the solar flares.

With a nominal transmitter frequency of $2.5 \mathrm{MHz}$, the ionospheric drift records during the daytime correspond to an altitude of about $100 \mathrm{~km}$ in the E-region. The corresponding altitude in the night-time is well within the F-region, as the E-region ionization is rapidly lost after sunset. Hence, the daytime observations of the drift speed correspond to the E-region heights and the night-time ones to the F-region.

During solar flares, solar radiation in the X-ray and extreme ultra violet region is increased. These radiations leads to increased ionization, particularly in the lower E-region. Thome and Wagner (1971) have shown that the E-region electron density during a flare may be enhanced by a factor of two or more. The increased electron density leads to increased ionospheric conductivity and currents. Therefore, the ground level geomagnetic field in the sunlit hemisphere is intensified for a short time during a flare. The effect is greatest at the sub-solar point and becomes smaller with increasing solar zenith angle. This effect is observed simultaneously with the optical flare on the solar disk; this kind of geomag- netic field variation is termed a geomagnetic solar flare effect, s.f.e. (e.g. Nagata and Fakushima, 1971). The effect is also called crochet. A detailed analysis of many such events has been given by Van Sabben (1961) and Nagata (1966). The crochet effect is excluded from magnetic disturbances because it is produced by a temporary increase in the solar radiation whereas magnetic disturbances are caused by corpuscular radiation from the sun and the solar wind. The s.f.e. is detectable, not only in the sunlit hemisphere but even in the dark hemisphere, though with much smaller magnitudes (Nagata, 1966). The height of the current associated with the crochet, near the sub solar point, is believed to be lower than that where the $S_{q}$ currents flow. The height of these current increases slightly with increasing distance from the sub solar point. The crochet effect found in the dark hemisphere is believed to be caused by a worldwide electric current, provoked by a sudden increase of conductivity in the sunlit hemisphere. The solar flare effect on the horizontal component of the geomagnetic field during the period of a strong counter electrojet is characterized by a negative crochet (decrease of the $H$ field) at an equatorial station and a positive crochet (increase of $H$ field) at a low latitude station outside the electrojet belt (Rastogi et al., 1975).

The plasma drift velocity depends on the neutral winds and the electric fields. Therefore, any change in drift velocity can be thought of as due to corresponding changes in the ionospheric neutral winds, or the electric fields, or both. Prölss (1982) has discussed mechanisms that can cause heating in the low-latitudes and a change in the wind pattern; these are ring current neutral particle injection, large-scale circulation and travelling atmospheric disturbances. Obviously, the time scales associated with these changes must be of the order of several hours and hence may not be invoked to account for the observed reduced drift speeds during the night-time. It is again emphasized that the night-time records correspond to the F-region drifts. Thus, the only other entity, which may be held responsible for the observed reduced drift speed, is the electric field. As has been noted above, the solar flares lead to increased ionization in the lower E-region in the sunlit hemisphere. The increased ionization leads to increased conductivity in the dynamo region. As the daytime dynamo is situated in the E-region, its efficiency in the generation of electric field, during a flare, is increased. The E-region field is transmitted to the F-region via the conducting lines of force. This mechanism is applicable latitudinally, in the same hemisphere. It is proposed that a transhemispherical coupling, arising due to the worldwide current system, could be effective during the flare time. This coupling should lead to the mapping of the E-region disturbance fields in the sunlit hemisphere to the F-region of the dark hemisphere. The direction of this field should be such as to oppose the normal field responsible for the horizontal drift. Effectively, the F-region electric fields must decrease to account for the reduced drift speeds during the night. At this stage, it is worth mentioning the numerical simulation work of Blanc and Richmond (1980) who discussed the possibility of modifications in the neutral wind system during magneti- 
cally disturbed period and the consequent modification of the dynamo. They suggested that the disturbance electric fields and currents act to reduce or reverse the normal $S_{q}$ current system over low equatorial latitudes. The recent observations of plasma depletions by Sinha et al. (2001) using multiwavelength imaging system from Kavalur (India), a low latitude station, on a magnetically disturbed night are equally relevant. The drift speed of these depletions was found to be far lower than on normal nights. Sinha et al. (2001) attributed the sluggish drift speed of plasma depletions to severe modification of the F-region dynamo electric fields affected by direct penetration of disturbance electric fields from high latitudes to low latitudes. However, their observations concern a magnetically disturbed period which would normally follow a day or two after the occurrence of a solar flare. Further, as has been noted earlier, magnetic disturbances are related to the solar wind rather than to solar flares.

With regard to the daytime case, although there is only one observation, it is clear that the reduction in drift speed is associated with the occurrence and onset of the flare, as was observed for the night-time cases. The results from Thumba (Misra, 1971) for the daytime E-region drifts, also report reduced speeds. The reduction in E-region drift speed could be attributed to increased ionization at lower heights and hence to the lower reflection heights corresponding to lower values of drift speeds.

Acknowledgements. Authors are grateful to the Head, Radio and Atmospheric Science Division, National Physical Laboratory, New Delhi for providing the Solar Geophysical data. The authors also thank Prof. R. K. Rai for fruitful discussions.

Topical Editor M. Lester thanks a referee for his help in evaluating this paper.

\section{References}

Blanc, M. and Richmond A. D.: The ionospheric disturbance dynamo, J. Geophys. Res., 85, 1669-1686, 1980.

Briggs, B. H. and Spencer, M.: Horizontal movement in the ionosphere, Rep. Prog. Phys., 17, 45-280, 1954.

Buonsanto, M. J.: Ionospheric storms-A review, Space Sci. Rev., 88, 563-601,1999.

Forbush, S. E. and Casaverde, M.: Equatorial Electrojet in Peru, Carnegie Institution of Washington, 620, 1961.

Mc Nish, A. G.: Terrestrial magnetic and ionspheric effects associated with bright chromospheric eruptions, Terr. Magn. Atms. elect., 42, 109-122, 1937.

Misra, R. K.: Studies on the ionospheric irregularities at low latitude, Ph.D. Thesis, Gujarat University, Ahmedabad, 1971.

Mitra, S. N.: A radio method of measuring winds in the ionosphere, Proc. Inst. Elect. Engrs., 96(III), 441-446, 1949.

Nagata, T.: Solar flare effect on the geomagnetic field, J. Geomag. Geoelec., 18, 197-219, 1966.

Nagata, T. and Fakushima, N.: Morphology of magnetic disturbance, Handbuch der Physik, vol. XLIX/3, Geophysik III, (Ed) Rawer, K., Springer-Verlag, Berlin, Heidelberg, New York, 1129, 1971.

Patel, V. P. and Chandra, H.: Ionospheric E-region drifts at Yamagawa during IQSY, J. Geomag. Geoelec., 32, 129-136, 1980.

Patel, V. P. and Chandra H.: Ionospheric E-region drifts at Sibizmir during 1970-75, Indian J. Radio \& Space Phys., 11, 187-189, 1982.

Prolss, G. W.: Perturbation of the low latitude upper atmosphere during magnetic substorm activity, J. Geophys. Res., 87, 52605266, 1982.

Rastogi, R. G., Chandra, H., and Misra, R. K.: Features of ionospheric drifts over the magnetic equator, Space Research, 12, 983-992, 1972.

Rastogi, R. G., Chandra, H., Singhal, K. P., Rai, R. K., Janve, A. V., and Kumar, V.: Simultaneous investigation of ionospheric Eregion irregularities at low latitude stations by spaced receiver technique, Indian J. Radio \& Space Phys, 7, 6-8, 1978.

Rastogi, R. G., Deshpande, M. R., and Sastri, N. S.: Solar flare effect in equatorial counter electrojet currents, Nature, 258, 218219, 1975.

Sinha, H. S. S., Rajesh, P. K., Misra, R. N., and Dutt, N.: Multiwavelength imaging observations of plasma depletions over Kavalur, India, Ann. Geophysicae, 19, 1-12, 2001.

Skinner, N. J., Lyon, A. J., and Wright, R. W.: Ionospheric drift measurement in the equatorial region, Proceedings of the International Conference on Ionosphere, (Ed) Stickland, A. C., Physical Society London, 301-309, 1963.

Thome, G. D. and Wagner, L. S.: Electron density enhancements in the E- and F-regions of the ionosphere during solar flares, J. Geophys. Res., 76, 6883-6895, 1971.

Van Sabben, D.: Ionospheric current systems of ten I.G.Y. - solar flare effects, J. Atmos. Terr. Phys., 22, 32-42, 1961.

Vyas, B. M.: Study of ionospheric irregularities of E- and F-region at a low latitude, Ph.D. Thesis, Mohan Lal Sukhadia University, Udaipur, India, 1989.

Vyas, G. D. and Chandra, H.: Ionospheric drift studies at equatorial electrojet region, Low Latitude Aeronomical Processes (Advances in Space Exploration), COSPAR Symp. Series, 8, 201203, 1979. 